\title{
GROUP INVERSE FOR THE BLOCK MATRIX WITH TWO IDENTICAL SUBBLOCKS OVER SKEW FIELDS*
}

\author{
JIEMEI ZHAO ${ }^{\dagger}$ AND CHANGJIANG BU§
}

\begin{abstract}
Let $K$ be a skew field and $K^{n \times n}$ be the set of all $n \times n$ matrices over $K$. The purpose of this paper is to give some necessary and sufficient conditions for the existence and the representations of the group inverse of the block matrix $\left(\begin{array}{cc}A & B \\ C & D\end{array}\right)$ under some conditions.
\end{abstract}

Key words. Skew fields, Block matrix, Group inverse.

AMS subject classifications. 15A09; 65F20.

1. Introduction. Let $K$ be a skew field, $\mathbb{C}$ be the complex number field, $K^{m \times n}$ be the set of all $m \times n$ matrices over $K$, and $I_{n}$ be the $n \times n$ identity matrix over $K$. For a matrix $A \in K^{n \times n}$, the matrix $X \in K^{n \times n}$ satisfying

$$
A^{k} X A=A^{k}, X A X=X, A X=X A
$$

is called the Drazin inverse of $A$ and is denoted by $X=A^{D}$, where $k$ is the index of $A$, i.e., the smallest non-negative integer such that $\operatorname{rank}\left(A^{k}\right)=\operatorname{rank}\left(A^{k+1}\right)$. We denote such a $k$ by $\operatorname{Ind}(A)$. It is well-known that $A^{D}$ exists and is unique (see [2]). If $\operatorname{Ind}(A)=1, A^{D}$ is also called the group inverse of $A$ and is denoted by $A^{\sharp}$. Then $A^{\sharp}$ exists if and only if $\operatorname{rank}(A)=\operatorname{rank}\left(A^{2}\right)($ see $[1,3,11-14,24,25,29])$. We denote $I-A A^{\sharp}$ by $A^{\pi}$.

The group inverse of block matrices has numerous applications in matrix theory, such as singular differential and difference equations, Markov chains, iterative methods and so on (see [12]-[14]). For instance, Y. Wei et al. studied the representation of the group inverse of a real singular Toeplitz matrix which arises in scientific computing and engineering (see [29]); in [25], S. Kirkland et al. investigated the representation of the group inverse of the Laplacian matrix of an undirected weighted graph $G$ on $n$ vertices; and in [24], G. Heinig studied the group inverse of the Sylvester transformation $\varphi(X)=A X-X B$, where $A \in \mathbb{C}^{m \times m}$ and $B \in \mathbb{C}^{n \times n}$; utilizing the theory of Drazin inverse, the differential equation $A x^{\prime}+B x=f$ is studied in linear systems,

*Received by the editors on June 2009. Accepted for publication on July 31, 2010 Handling Editors: Roger A. Horn and Fuzhen Zhang.

${ }^{\dagger}$ College of Automation, Harbin Engineering University, Harbin 150001, P. R. China. (zhaojiemei500@163.com). Supported by the NSFH, No.159110120002.

${ }^{\ddagger}$ Dept. of Applied Math., College of Science, Harbin Engineering University, Harbin 150001, P. R. China. ${ }^{\S}$ Corresponding author. Email: buchangjiang@hrbeu.edu.cn 
where $A, B$ are both $n \times n$ singular matrices and $f$ is a vector-valued function (see [13]); in [3], R. Bru et al. gave the general explicit solution which is obtained when the symmetric singular linear control system satisfies the regularity condition.

In 1979, S. Campbell and C. Meyer proposed an open problem to find an explicit representation for the Drazin inverse of a $2 \times 2$ block matrix $\left(\begin{array}{ll}A & B \\ C & D\end{array}\right)$, where the blocks $A$ and $D$ are supposed to be square matrices but their sizes need not be the same (see [12]). A simplified problem to find an explicit representation of the Drazin (group) inverse for block matrix $\left(\begin{array}{cc}A & B \\ C & O\end{array}\right)$ ( $A$ is square, $O$ is square null matrix) was proposed by S. Campbell in 1983. This open problem was motivated in hoping to find general expressions for the solutions of the second-order system of the differential equations

$$
E x^{\prime \prime}(t)+F x^{\prime}(t)+G x(t)=0(t \geq 0),
$$

where $E$ is a singular matrix. Detailed discussions of the importance of the problem can be found in [11]. Until now, both problems have not been resolved. However, there have been some studies about the representations of the Drazin(group) inverse under certain conditions (see [4-10, 15-23, 26, 27]). For example, the formulae of Drazin(group) inverse of an operator matrix under some conditions are investigated in [18] and [19]. The representations for the Drazin(group) inverse of the sum of several matrices are given in [15] and [17]. In particular, J. Chen et al. [15] presented the representation of the group inverse of $M=P+Q+S$, where $P Q=Q P=O$, $P S=S Q=O, P^{\sharp}$ and $Q^{\sharp}$ exist. Some conditions for the representations of group inverse for block matrix $\left(\begin{array}{ll}A & B \\ C & D\end{array}\right)$ are listed below:

(i) $A^{\sharp}$ and $\left(D-C A^{\sharp} B\right)^{\sharp}$ exist [18].

(ii) $D=O, A=B=I_{d}, I_{d}, C \in \mathbb{C}^{d \times d}[10]$.

(iii) $D=O, A, B, C \in\left\{P, P^{*}, P P^{*}\right\}, P \in \mathbb{C}^{n \times n}, P^{2}=P, P^{*}$ is the conjugate transpose of $P[9]$.

(iv) $C=O, A$ and $D$ are square matrices over $K[8]$.

(v) $D=O, A=B=A^{2}, A, C \in K^{n \times n}[4]$.

(vi) $D=O, A=B, \operatorname{rank}(C) \geq \operatorname{rank}(A), A, C \in K^{n \times n}[5]$.

(vii) $A$ is invertible and $\left(D-C A^{-1} B\right)^{\sharp}$ exists; $D$ is invertible and $\left(A-B D^{-1} C\right)^{\sharp}$ exists; $B$ or $C$ is invertible, where $A, B, C \in K^{n \times n}[6]$.

(viii) $D=O, A=c_{1} B+c_{2} C$, where non-zero elements $c_{1}$ and $c_{2}$ are in the center of $K ; D=O, A=B^{k} C^{l}$, where $k$ and $l$ are positive integers [7].

In order to further solve the problem proposed in [11], in this paper, we give the sufficient conditions or the necessary and sufficient conditions for the existence and the representations of the group inverse for block matrix $\left(\begin{array}{cc}A & B \\ C & D\end{array}\right)\left(A, B \in K^{n \times n}\right)$ when $A$ and $B$ satisfy one of the following conditions:

(1) $B^{\sharp}$ and $\left(B^{\pi} A\right)^{\sharp}$ exist; 
(2) $B^{\sharp}$ and $\left(A B^{\pi}\right)^{\sharp}$ exist;

(3) $B^{\sharp}$ exists and $B A B^{\pi}=O$;

(4) $B^{\sharp}$ exists and $B^{\pi} A B=O$.

\section{Some Lemmas.}

Lemma 2.1. Let $A \in K^{n \times n}$. Then $A$ has a group inverse if and only if there exist nonsingular matrices $P \in K^{n \times n}$ and $A_{1} \in K^{r \times r}$ such that $A=P\left(\begin{array}{cc}A_{1} & O \\ O & O\end{array}\right) P^{-1}$ and $A^{\sharp}=P\left(\begin{array}{cc}A_{1} \sharp & O \\ O & O\end{array}\right) P^{-1}$, where $\operatorname{rank}(A)=r$.

Proof. Theorem 2.2.2 of [28] holds over the complex number field and also over skew fields. $\square$

Lemma 2.2. [7] Let $A, G \in K^{n \times n}, \operatorname{Ind}(A)=k$. Then $G=A^{D}$ if and only if

$$
A^{k} G A=A^{k}, \quad A G=G A, \quad \operatorname{rank}(G) \leq \operatorname{rank}\left(A^{k}\right) .
$$

Lemma 2.3. Let $M=\left(\begin{array}{cc}A & B \\ B & O\end{array}\right), S=B^{\pi} A B^{\pi}, A, B \in K^{n \times n}$.

(i) If $B^{\sharp}$ and $\left(B^{\pi} A\right)^{\sharp}$ exist, then $S^{\sharp}$ and $M^{\sharp}$ exist.

(ii) If $B^{\sharp}$ exists and $B A B^{\pi}=O$, then $M^{\sharp}$ exists if and only if $\left(A B^{\pi}\right)^{\sharp}$ exists.

Proof. Suppose $\operatorname{rank}(B)=r$. Applying Lemma 2.1, there exist invertible matrices $P \in K^{n \times n}$ and $B_{1} \in K^{r \times r}$ such that

$$
B=P\left(\begin{array}{cc}
B_{1} & O \\
O & O
\end{array}\right) P^{-1} \quad \text { and } \quad B^{\sharp}=P\left(\begin{array}{cc}
B_{1}^{-1} & O \\
O & O
\end{array}\right) P^{-1} .
$$

Let $A=P\left(\begin{array}{cc}A_{1} & A_{2} \\ A_{3} & A_{4}\end{array}\right) P^{-1}$, where $A_{1} \in K^{r \times r}, A_{2} \in K^{r \times(n-r)}, A_{3} \in K^{(n-r) \times r}$ and $A_{4} \in K^{(n-r) \times(n-r)}$.

(i) Because $\left(B^{\pi} A\right)^{\sharp}$ exists, we have $\operatorname{rank}\left(B^{\pi} A\right)=\operatorname{rank}\left(B^{\pi} A\right)^{2}$, that is,

$$
\operatorname{rank}\left(A_{3} \quad A_{4}\right)=\operatorname{rank}\left(A_{4} A_{3} \quad A_{4}^{2}\right)
$$

Since $\operatorname{rank}\left(A_{3} A_{4}\right)=\operatorname{rank}\left(A_{4}\left(A_{3} A_{4}\right)\right) \leq \operatorname{rank}\left(A_{4}\right)$ and $\operatorname{rank}\left(A_{3} A_{4}\right) \geq \operatorname{rank}\left(A_{4}\right)$, we have $\operatorname{rank}\left(A_{3} A_{4}\right)=\operatorname{rank}\left(A_{4}\right)$.

So there exists a matrix $X \in K^{(n-r) \times r}$ such that $A_{3}=A_{4} X$. 
66

Hence, $\operatorname{rank}\left(A_{4}\right)=\operatorname{rank}\left(A_{4}^{2}\right)$, i.e., $A_{4}^{\sharp}$ exists. Noting that

$$
\begin{aligned}
S & =B^{\pi} A B^{\pi} \\
& =P\left(\begin{array}{cc}
O & O \\
O & I_{n-r}
\end{array}\right)\left(\begin{array}{cc}
A_{1} & A_{2} \\
A_{3} & A_{4}
\end{array}\right)\left(\begin{array}{cc}
O & O \\
O & I_{n-r}
\end{array}\right) P^{-1} \\
& =P\left(\begin{array}{cc}
O & O \\
O & A_{4}
\end{array}\right) P^{-1},
\end{aligned}
$$

we see that $S^{\sharp}$ exists. Since

$$
\begin{aligned}
\operatorname{rank}(M) & =\operatorname{rank}\left(\begin{array}{cccc}
A_{1} & A_{2} & B_{1} & O \\
A_{3} & A_{4} & O & O \\
B_{1} & O & O & O \\
O & O & O & O
\end{array}\right)=\operatorname{rank}\left(\begin{array}{cccc}
O & O & B_{1} & O \\
O & A_{4} & O & O \\
B_{1} & O & O & O \\
O & O & O & O
\end{array}\right) \\
& =2 r+\operatorname{rank}\left(A_{4}\right)
\end{aligned}
$$

and

$$
\begin{aligned}
\operatorname{rank}\left(M^{2}\right) & =\operatorname{rank}\left(\begin{array}{cc}
A^{2}+B^{2} & A B \\
B A & B^{2}
\end{array}\right)=\operatorname{rank}\left(\begin{array}{ccc}
A^{2}-A B B^{\sharp} A+B^{2} & O \\
O & B^{2}
\end{array}\right) \\
& =\operatorname{rank}\left(\begin{array}{cccc}
B_{1}^{2}+A_{2} A_{3} & A_{2} A_{4} & O & O \\
A_{4} A_{3} & A_{4}^{2} & O & O \\
O & O & B_{1}^{2} & O \\
O & O & O & O
\end{array}\right)
\end{aligned}
$$

by $A_{3}=A_{4} X$, we get

$$
\operatorname{rank}\left(M^{2}\right)=\operatorname{rank}\left(\begin{array}{cccc}
B_{1}^{2} & O & O & O \\
O & A_{4}^{2} & O & O \\
O & O & B_{1}^{2} & O \\
O & O & O & O
\end{array}\right)=2 r+\operatorname{rank}\left(A_{4}^{2}\right),
$$

and

$$
\operatorname{rank}(M)=\operatorname{rank}\left(M^{2}\right),
$$

i.e., $M^{\sharp}$ exists.

(ii) If $B A B^{\pi}=O$, then $A_{2}=O$, thus $A B^{\pi}=P\left(\begin{array}{cc}O & O \\ O & A_{4}\end{array}\right) P^{-1}$,

$$
\operatorname{rank}(M)=\operatorname{rank}\left(\begin{array}{cccc}
A_{1} & O & B_{1} & O \\
A_{3} & A_{4} & O & O \\
B_{1} & O & O & O \\
O & O & O & O
\end{array}\right)=\operatorname{rank}\left(\begin{array}{cccc}
O & O & B_{1} & O \\
O & A_{4} & O & O \\
B_{1} & O & O & O \\
O & O & O & O
\end{array}\right)
$$




$$
=2 r+\operatorname{rank}\left(A_{4}\right)
$$

and

$$
\begin{aligned}
& \operatorname{rank}\left(M^{2}\right)=\operatorname{rank}\left(\begin{array}{cc}
A^{2}+B^{2} & A B \\
B A & B^{2}
\end{array}\right)=\operatorname{rank}\left(\begin{array}{cc}
A^{2}-A B B^{\sharp} A+B^{2} & O \\
O & B
\end{array}\right) \\
& =\operatorname{rank}\left(\begin{array}{cccc}
B_{1}^{2} & O & O & O \\
A_{4} A_{3} & A_{4}^{2} & O & O \\
O & O & B_{1}^{2} & O \\
O & O & O & O
\end{array}\right)=\operatorname{rank}\left(\begin{array}{cccc}
B_{1}^{2} & O & O & O \\
O & A_{4}^{2} & O & O \\
O & O & B_{1}^{2} & O \\
O & O & O & O
\end{array}\right) \\
& =2 r+\operatorname{rank}\left(A_{4}^{2}\right) \text {. }
\end{aligned}
$$

As $\left(A B^{\pi}\right)^{\sharp}$ exists, we get $\operatorname{rank}\left(A_{4}\right)=\operatorname{rank}\left(A_{4}^{2}\right) . \operatorname{Thus} \operatorname{rank}(M)=\operatorname{rank}\left(M^{2}\right)$; that is, $M^{\sharp}$ exists, so the "if" part holds. Now we prove the "only if" part.

Since $M^{\sharp}$ exists if and only if $\operatorname{rank}(M)=\operatorname{rank}\left(M^{2}\right), \operatorname{rank}\left(A_{4}\right)=\operatorname{rank}\left(A_{4}^{2}\right)$, i.e., $\left(A B^{\pi}\right)^{\sharp}$ exists.

Lemma 2.4. Let $A, B \in K^{n \times n}, S=B^{\pi} A B^{\pi}$. Suppose $B^{\sharp}$ and $\left(B^{\pi} A\right)^{\sharp}$ exist. Then $S^{\sharp}$ exists and the following conclusions hold:

(i) $B^{\pi} A S^{\sharp} A=B^{\pi} A$;

(ii) $B^{\pi} A S^{\sharp}=S^{\sharp} A B^{\pi}$;

(iii) $B S^{\sharp}=S^{\sharp} B=B^{\sharp} S^{\sharp}=S^{\sharp} B^{\sharp}=O$.

Proof. Suppose $\operatorname{rank}(B)=r$. Applying Lemma 2.1, there exist invertible matrices $P \in K^{n \times n}$ and $B_{1} \in K^{r \times r}$ such that $B=P\left(\begin{array}{cc}B_{1} & O \\ O & O\end{array}\right) P^{-1}$ and

$$
B^{\sharp}=P\left(\begin{array}{cc}
B_{1}^{-1} & O \\
O & O
\end{array}\right) P^{-1} .
$$

Let $A=P\left(\begin{array}{cc}A_{1} & A_{2} \\ A_{3} & A_{4}\end{array}\right) P^{-1}$, where $A_{1} \in K^{r \times r}, A_{2} \in K^{r \times(n-r)}, A_{3} \in K^{(n-r) \times r}$ and $A_{4} \in K^{(n-r) \times(n-r)}$.

From Lemma $2.3(i)$ and the proof of Lemma $2.3(i)$, we get $S^{\sharp}$ exists and

$$
S^{\sharp}=P\left(\begin{array}{cc}
O & O \\
O & A_{4}^{\sharp}
\end{array}\right) P^{-1} .
$$

(i) $B^{\pi} A S^{\sharp} A=P\left(\begin{array}{cc}O & O \\ A_{3} & A_{4}\end{array}\right)\left(\begin{array}{cc}O & O \\ O & A_{4}^{\sharp}\end{array}\right)\left(\begin{array}{ll}A_{1} & A_{2} \\ A_{3} & A_{4}\end{array}\right) P^{-1}$ 
68

$$
=P\left(\begin{array}{cc}
O & O \\
A_{4} A_{4}^{\sharp} A_{3} & A_{4}
\end{array}\right) P^{-1} .
$$

By $A_{3}=A_{4} X, X \in K^{(n-r) \times r}$, we have

$$
B^{\pi} A S^{\sharp} A=P\left(\begin{array}{cc}
O & O \\
A_{3} & A_{4}
\end{array}\right) P^{-1}=B^{\pi} A .
$$

(ii) $B^{\pi} A S^{\sharp}=P\left(\begin{array}{cc}O & O \\ O & A_{4} A_{4}^{\sharp}\end{array}\right) P^{-1}=S^{\sharp} A B^{\pi}$.

(iii) $B S^{\sharp}=P\left(\begin{array}{cc}B_{1} & O \\ O & O\end{array}\right)\left(\begin{array}{cc}O & O \\ O & A_{4}^{\sharp}\end{array}\right) P^{-1}=O$.

Similarly, we obtain $S^{\sharp} B=B^{\sharp} S^{\sharp}=S^{\sharp} B^{\sharp}=O$. $\square$

Lemma 2.5. Let $A, B \in K^{n \times n}$. If $B A B^{\pi}=O, B^{\sharp}$ and $\left(A B^{\pi}\right)^{\sharp}$ exist, then

(a) $A\left(A B^{\pi}\right)^{\sharp} A B^{\pi}=A B^{\pi}$;

(b) $A\left(A B^{\pi}\right)^{\sharp}=\left(A B^{\pi}\right)^{\sharp} A B^{\pi}$;

(c) $B^{\sharp} A B^{\pi}=O,\left(A B^{\pi}\right)^{\sharp} B=O, B\left(A B^{\pi}\right)^{\sharp}=O$.

Proof. Suppose $\operatorname{rank}(B)=r$. Applying Lemma 2.1, there exist invertible matrices $P \in K^{n \times n}$ and $B_{1} \in K^{r \times r}$ such that

$$
B=P\left(\begin{array}{cc}
B_{1} & O \\
O & O
\end{array}\right) P^{-1} \text { and } B^{\sharp}=P\left(\begin{array}{cc}
B_{1}^{-1} & O \\
O & O
\end{array}\right) P^{-1} \text {. }
$$

Let $A=P\left(\begin{array}{cc}A_{1} & A_{2} \\ A_{3} & A_{4}\end{array}\right) P^{-1}$, where $A_{1} \in K^{r \times r}, A_{2} \in K^{r \times(n-r)}, A_{3} \in K^{(n-r) \times r}$ and $A_{4} \in K^{(n-r) \times(n-r)}$. By $B A B^{\pi}=O$, we get $A=P\left(\begin{array}{cc}A_{1} & O \\ A_{3} & A_{4}\end{array}\right) P^{-1}$. Then

(a) $A\left(A B^{\pi}\right)^{\sharp} A B^{\pi}=P\left(\begin{array}{cc}A_{1} & O \\ A_{3} & A_{4}\end{array}\right)\left(\begin{array}{cc}O & O \\ O & A_{4}^{\sharp}\end{array}\right)\left(\begin{array}{cc}O & O \\ O & A_{4}\end{array}\right) P^{-1}$

$$
=P\left(\begin{array}{cc}
O & O \\
O & A_{4}
\end{array}\right) P^{-1}=A B^{\pi} .
$$

(b) $A\left(A B^{\pi}\right)^{\sharp}=P\left(\begin{array}{cc}A_{1} & O \\ A_{3} & A_{4}\end{array}\right)\left(\begin{array}{cc}O & O \\ O & A_{4}^{\sharp}\end{array}\right) P^{-1}=P\left(\begin{array}{cc}O & O \\ O & A_{4}^{\sharp} A_{4}\end{array}\right) P^{-1}$

$$
=\left(A B^{\pi}\right)^{\sharp} A B^{\pi} \text {. }
$$

(c) $B^{\sharp} A B^{\pi}=P\left(\begin{array}{cc}B_{1}^{-1} & O \\ O & O\end{array}\right)\left(\begin{array}{cc}O & O \\ O & A_{4}\end{array}\right) P^{-1}=O$.

Similarly, we have $\left(A B^{\pi}\right)^{\sharp} B=O, B\left(A B^{\pi}\right)^{\sharp}=O$. $\square$ 


\section{Conclusions.}

Theorem 3.1. Let $M=\left(\begin{array}{cc}A & B \\ B & O\end{array}\right)$, where $A, B \in K^{n \times n}$. Suppose $B^{\sharp}$ and $\left(B^{\pi} A\right)^{\sharp}$ exist. Then $M^{\sharp}$ exists and

$$
M^{\sharp}=\left(\begin{array}{cc}
U_{11} & U_{12} \\
U_{21} & U_{22}
\end{array}\right),
$$

where

$$
\begin{aligned}
U_{11} & =S^{\sharp}+\left(S^{\sharp} A-I\right) B B^{\sharp} A B^{\pi} A S^{\sharp}-\left(S^{\sharp} A-I\right) B B^{\sharp} A B^{\pi} ; \\
U_{12} & =B^{\sharp}-S^{\sharp} A B^{\sharp} ; \\
U_{21} & =B^{\sharp}-B^{\sharp} A S^{\sharp}+B^{\sharp} A\left(S^{\sharp} A-I\right) B B^{\sharp} A B^{\pi}-B^{\sharp} A\left(S^{\sharp} A-I\right) B B^{\sharp} A B^{\pi} A S^{\sharp} ; \\
U_{22} & =B^{\sharp} A S^{\sharp} A B^{\sharp}-B^{\sharp} A B^{\sharp} ; \\
S & =B^{\pi} A B^{\pi} .
\end{aligned}
$$

Proof. The existence of $M^{\sharp}$ and $S^{\sharp}$ have been given in Lemma $2.3(i)$.

Let $X=\left(\begin{array}{cc}U_{11} & U_{12} \\ U_{21} & U_{22}\end{array}\right)$. Then

$$
M X=\left(\begin{array}{cc}
A U_{11}+B U_{21} & A U_{12}+B U_{22} \\
B U_{11} & B U_{12}
\end{array}\right) \text { and } X M=\left(\begin{array}{cc}
U_{11} A+U_{12} B & U_{11} B \\
U_{21} A+U_{22} B & U_{21} B
\end{array}\right) .
$$

We prove $X=M^{\sharp}$. Applying (i)-(iii) of Lemma 2.4, we get the following identities:

$$
\begin{aligned}
A U_{11}+B U_{21}= & A S^{\sharp}+A\left(S^{\sharp} A-I\right) B B^{\sharp} A B^{\pi} A S^{\sharp}-A\left(S^{\sharp} A-I\right) B B^{\sharp} A B^{\pi}+B B^{\sharp} \\
& -B B^{\sharp} A S^{\sharp}+B B^{\sharp} A\left(S^{\sharp} A-I\right) B B^{\sharp} A B^{\pi}-B B^{\sharp} A\left(S^{\sharp} A-I\right) B B^{\sharp} A B^{\pi} A S^{\sharp} \\
= & B B^{\sharp}+B^{\pi} A S^{\sharp}+B^{\pi} A\left(S^{\sharp} A-I\right) B B^{\sharp} A B^{\pi} A S^{\sharp}-B^{\pi} A\left(S^{\sharp} A-I\right) B B^{\sharp} A B^{\pi} \\
\stackrel{(i)}{=} & B^{\pi} A S^{\sharp}+B B^{\sharp},
\end{aligned}
$$

and

$$
\begin{aligned}
U_{11} A+U_{12} B= & S^{\sharp} A+\left(S^{\sharp} A-I\right) B B^{\sharp} A B^{\pi} A S^{\sharp} A-\left(S^{\sharp} A-I\right) B B^{\sharp} A B^{\pi} A+B B^{\sharp} \\
& -S^{\sharp} A B B^{\sharp} \\
\stackrel{(i)}{=} & S^{\sharp} A B^{\pi}+B B^{\sharp} \\
\stackrel{(i i)}{=} & B^{\pi} A S^{\sharp}+B B^{\sharp} .
\end{aligned}
$$


Hence,

$$
\begin{aligned}
A U_{11}+B U_{21} & =U_{11} A+U_{12} B . \\
A U_{12}+B U_{22} & =A B^{\sharp}-B^{\pi} A S^{\sharp} A B^{\sharp}-B B^{\sharp} A B^{\sharp} \\
& \stackrel{(i)}{=} A B^{\sharp}-B^{\pi} A B^{\sharp}-B B^{\sharp} A B^{\sharp} \\
& =O, \\
U_{11} B & =S^{\sharp} B+\left(S^{\sharp} A-I\right) B B^{\sharp} A B^{\pi} A S^{\sharp} B-\left(S^{\sharp} A-I\right) B B^{\sharp} A B^{\pi} B \\
& =S^{\sharp} B+\left(S^{\sharp} A-I\right) B B^{\sharp} A B^{\pi} A S^{\sharp} B \\
& \stackrel{(i i i)}{=} O .
\end{aligned}
$$

Therefore, $A U_{12}+B U_{22}=U_{11} B$. Similarly, we can get

$$
\begin{aligned}
& B U_{11}=U_{21} A+U_{22} B=B B^{\sharp} A B^{\pi}\left(I-A S^{\sharp}\right), \\
& B U_{12}=U_{21} B=B B^{\sharp} .
\end{aligned}
$$

Consequently, $M X=X M=\left(\begin{array}{cc}B B^{\sharp}+B^{\pi} A S^{\sharp} & O \\ B B^{\sharp} A B^{\pi}\left(I-A S^{\sharp}\right) & B B^{\sharp}\end{array}\right)$.

It is easy to compute

$$
M X M=\left(\begin{array}{cc}
B B^{\sharp}+B^{\pi} A S^{\sharp} & O \\
B B^{\sharp} A B^{\pi}\left(I-A S^{\sharp}\right) & B B^{\sharp}
\end{array}\right)\left(\begin{array}{cc}
A & B \\
B & O
\end{array}\right)=\left(\begin{array}{cc}
A & B \\
B & O
\end{array}\right)=M .
$$

Suppose $\operatorname{rank}(B)=r$. By Lemma 2.1, there exist invertible matrices $P \in K^{n \times n}$ and $B_{1} \in K^{r \times r}$ such that $B=P\left(\begin{array}{cc}B_{1} & O \\ O & O\end{array}\right) P^{-1}$ and $B^{\sharp}=P\left(\begin{array}{cc}B_{1}^{-1} & O \\ O & O\end{array}\right) P^{-1}$.

Let $A=P\left(\begin{array}{cc}A_{1} & A_{2} \\ A_{3} & A_{4}\end{array}\right) P^{-1}, \quad$ where $A_{1} \in K^{r \times r}, A_{2} \in K^{r \times(n-r)}, A_{3} \in$ $K^{(n-r) \times r}$ and $A_{4} \in K^{(n-r) \times(n-r)}$.

$$
\begin{aligned}
\operatorname{rank}(X) & =\operatorname{rank}\left(\begin{array}{cc}
U_{11} & U_{12} \\
U_{21} & U_{22}
\end{array}\right) \\
& =\operatorname{rank}\left(\begin{array}{c}
S^{\sharp}+\left(S^{\sharp} A-I\right) B B^{\sharp} A B^{\pi} A S^{\sharp}-\left(S^{\sharp} A-I\right) B B^{\sharp} A B^{\pi} \\
B^{\sharp} \\
\end{array}\right. \\
& =\operatorname{rank}\left(\begin{array}{cc}
S^{\sharp} & B^{\sharp}-S^{\sharp} A B^{\sharp} \\
B^{\sharp} & O
\end{array}\right)=\operatorname{rank}\left(\begin{array}{cccc}
O & O & B_{1}^{-1} & O \\
O & A_{4}^{\sharp} & O & O \\
B_{1}^{-1} & O & O & O \\
O & O & O & O
\end{array}\right) \\
& =2 r+\operatorname{rank}\left(A_{4}^{\sharp}\right)=2 r+\operatorname{rank}\left(A_{4}\right)=\operatorname{rank}(M) .
\end{aligned}
$$

From Lemma 2.2 , we get $X=M^{\sharp}$. 
Now we present an example to show the representation of the group inverse for a block matrix over the real quaternion skew field.

EXAMPLE 3.2. Let the real quaternion skew field $K=\{a+b i+c j+d k\}$, where $a, b, c$ and $d$ are real numbers. Let $M=\left(\begin{array}{cc}A & B \\ B & O\end{array}\right)$, where $A=\left(\begin{array}{cc}0 & 0 \\ 0 & k\end{array}\right)$ and $B=\left(\begin{array}{cc}i & j \\ 0 & 0\end{array}\right)$.

By computation, $B^{\sharp}$ and $\left(B^{\pi} A\right)^{\sharp}$ exist, and $B^{\sharp}=\left(\begin{array}{rr}-i & -j \\ 0 & 0\end{array}\right)$,

$S^{\sharp}=\left(\begin{array}{rr}0 & 1 \\ 0 & -k\end{array}\right)$.

By Theorem 3.1, $M^{\sharp}$ exists and $U_{11}=\left(\begin{array}{rr}0 & 1 \\ 0 & -k\end{array}\right), U_{12}=\left(\begin{array}{rr}-i & -j \\ 0 & 0\end{array}\right), U_{21}=$ $\left(\begin{array}{rr}-i & 0 \\ 0 & 0\end{array}\right), U_{22}=\left(\begin{array}{ll}0 & 0 \\ 0 & 0\end{array}\right)$, so $M^{\sharp}=\left(\begin{array}{rrrr}0 & 1 & -i & -j \\ 0 & -k & 0 & 0 \\ -i & 0 & 0 & 0 \\ 0 & 0 & 0 & 0\end{array}\right)$.

Theorem 3.3. Let $M=\left(\begin{array}{cc}A & B \\ B & O\end{array}\right)$, where $A, B \in K^{n \times n}$. Suppose $B^{\sharp}$ and $\left(A B^{\pi}\right)^{\sharp}$ exist. Then $M^{\sharp}$ exists and

$$
M^{\sharp}=\left(\begin{array}{ll}
U_{11} & U_{12} \\
U_{21} & U_{22}
\end{array}\right),
$$

where

$$
\begin{aligned}
U_{11} & =S^{\sharp}+S^{\sharp} A B^{\pi} A B B^{\sharp}\left(A S^{\sharp}-I\right)-B^{\pi} A B B^{\sharp}\left(A S^{\sharp}-I\right) ; \\
U_{12} & =B^{\sharp}-S^{\sharp} A B^{\sharp}-S^{\sharp} A B^{\pi} A B B^{\sharp}\left(A S^{\sharp}-I\right) A B^{\sharp}+B^{\pi} A B B^{\sharp}\left(I-A S^{\sharp}\right) A B^{\sharp} ; \\
U_{21} & =B^{\sharp}-B^{\sharp} A S^{\sharp} ; \\
U_{22} & =B^{\sharp} A S^{\sharp} A B^{\sharp}-B^{\sharp} A B^{\sharp} ; \\
S & =B^{\pi} A B^{\pi} .
\end{aligned}
$$

Proof. The proof is similar to that of Theorem 3.1.

Theorem 3.4. Let $M=\left(\begin{array}{cc}A & B \\ B & O\end{array}\right)$, where $A, B \in K^{n \times n}$. Suppose $B^{\sharp}$ exists and $B A B^{\pi}=O$. Then

(i) $M^{\sharp}$ exists if and only if $\left(A B^{\pi}\right)^{\sharp}$ exists. 
(ii) If $M^{\sharp}$ exists, then

$$
M^{\sharp}=\left(\begin{array}{cc}
U & V \\
B^{\sharp} & -B^{\sharp} A B^{\sharp}
\end{array}\right),
$$

where

$$
\begin{aligned}
& U=B^{\pi} A\left(B^{\sharp}\right)^{2}-\left(A B^{\pi}\right)^{\sharp} A B^{\pi} A\left(B^{\sharp}\right)^{2}+\left(A B^{\pi}\right)^{\sharp} ; \\
& V=-B^{\pi} A\left(B^{\sharp}\right)^{2} A B^{\sharp}+\left(A B^{\pi}\right)^{\sharp} A B^{\pi} A\left(B^{\sharp}\right)^{2} A B^{\sharp}-\left(A B^{\pi}\right)^{\sharp} A B^{\sharp}+B^{\sharp} .
\end{aligned}
$$

Proof. (i) The existence of $M^{\sharp}$ has been given in Lemma 2.3 (ii).

(ii) Let $X=\left(\begin{array}{cc}U & V \\ B^{\sharp} & -B^{\sharp} A B^{\sharp}\end{array}\right)$. Then

$$
M X=\left(\begin{array}{cc}
A U+B B^{\sharp} & A V-B B^{\sharp} A B^{\sharp} \\
B U & B V
\end{array}\right) \text { and } X M=\left(\begin{array}{cc}
U A+V B & U B \\
B^{\sharp} A B^{\pi} & B^{\sharp} B
\end{array}\right) .
$$

We prove $M X=X M$ by Lemma 2.5 .

$$
\begin{aligned}
A U+B B^{\sharp} & =A B^{\pi} A\left(B^{\sharp}\right)^{2}-A\left(A B^{\pi}\right)^{\sharp} A B^{\pi} A\left(B^{\sharp}\right)^{2}+A\left(A B^{\pi}\right)^{\sharp}+B B^{\sharp} \\
& \stackrel{(a)}{=} A B^{\pi} A\left(B^{\sharp}\right)^{2}-A B^{\pi} A\left(B^{\sharp}\right)^{2}+A\left(A B^{\pi}\right)^{\sharp}+B B^{\sharp} \\
& =A\left(A B^{\pi}\right)^{\sharp}+B B^{\sharp} . \\
U A+V B & =B^{\pi} A\left(B^{\sharp}\right)^{2} A B^{\pi}-\left(A B^{\pi}\right)^{\sharp} A B^{\pi} A\left(B^{\sharp}\right)^{2} A B^{\pi}+\left(A B^{\pi}\right)^{\sharp} A B^{\pi}+B^{\sharp} B \\
& \stackrel{(c)}{=}\left(A B^{\pi}\right)^{\sharp} A B^{\pi}+B^{\sharp} B \\
& \stackrel{(b)}{=} A\left(A B^{\pi}\right)^{\sharp}+B B^{\sharp} .
\end{aligned}
$$

Thus,

$$
\begin{aligned}
A U+B B^{\sharp}= & U A+V B . \\
A V-B B^{\sharp} A B^{\sharp}= & -A B^{\pi} A\left(B^{\sharp}\right)^{2} A B^{\sharp}+A\left(A B^{\pi}\right)^{\sharp} A B^{\pi} A\left(B^{\sharp}\right)^{2} A B^{\sharp}-A\left(A B^{\pi}\right)^{\sharp} A B^{\sharp} \\
& +A B^{\sharp}-B B^{\sharp} A B^{\sharp} \\
\stackrel{(a)}{=} & -A\left(A B^{\pi}\right)^{\sharp} A B^{\sharp}+B^{\pi} A B^{\sharp} \\
U B= & B^{\pi} A B^{\sharp}-\left(A B^{\pi}\right)^{\sharp} A B^{\pi} A B^{\sharp}+\left(A B^{\pi}\right)^{\sharp} B \\
\stackrel{(b)}{=} & B^{\pi} A B^{\sharp}-A\left(A B^{\pi}\right)^{\sharp} A B^{\sharp}+\left(A B^{\pi}\right)^{\sharp} B \\
& \stackrel{(c)}{=}-A\left(A B^{\pi}\right)^{\sharp} A B^{\sharp}+B^{\pi} A B^{\sharp} .
\end{aligned}
$$

Hence, $A V-B B^{\sharp} A B^{\sharp}=U B$. Similarly, we can obtain

$$
B U=B^{\sharp} A B^{\pi}=O, B V=B^{\sharp} B .
$$


Consequently,

$$
M X=X M=\left(\begin{array}{cc}
A\left(A B^{\pi}\right)^{\sharp}+B B^{\sharp} & -A\left(A B^{\pi}\right)^{\sharp} A B^{\sharp}+B^{\pi} A B^{\sharp} \\
O & B B^{\sharp}
\end{array}\right) .
$$

Applying Lemma 2.5, it is easy to compute

$$
\begin{aligned}
M X M & =\left(\begin{array}{cc}
A\left(A B^{\pi}\right)^{\sharp}+B B^{\sharp} & -A\left(A B^{\pi}\right)^{\sharp} A B^{\sharp}+B^{\pi} A B^{\sharp} \\
O & B B^{\sharp}
\end{array}\right)\left(\begin{array}{cc}
A & B \\
B & O
\end{array}\right) \\
& =\left(\begin{array}{cc}
A & B \\
B & O
\end{array}\right)=M .
\end{aligned}
$$

Suppose $\operatorname{rank}(B)=r$. Applying Lemma 2.1, there exist invertible matrices $P \in$ $K^{n \times n}$ and $B_{1} \in K^{r \times r}$ such that

$$
B=P\left(\begin{array}{cc}
B_{1} & O \\
O & O
\end{array}\right) P^{-1} \text { and } B^{\sharp}=P\left(\begin{array}{cc}
B_{1}^{-1} & O \\
O & O
\end{array}\right) P^{-1} .
$$

Let $A=P\left(\begin{array}{cc}A_{1} & A_{2} \\ A_{3} & A_{4}\end{array}\right) P^{-1}$, where $A_{1} \in K^{r \times r}, A_{2} \in K^{r \times(n-r)}, A_{3} \in K^{(n-r) \times r}$, and $A_{4} \in K^{(n-r) \times(n-r)}$. Then

$$
\begin{aligned}
& \operatorname{rank}(X)=\operatorname{rank}\left(\begin{array}{cc}
U & V \\
B^{\sharp} & -B^{\sharp} A B^{\sharp}
\end{array}\right)=\operatorname{rank}\left(\begin{array}{cc}
U & B^{\sharp} \\
B^{\sharp} & O
\end{array}\right) \\
& =\operatorname{rank}\left(\begin{array}{cc}
\left(A B^{\pi}\right)^{\sharp} & B^{\sharp} \\
B^{\sharp} & O
\end{array}\right)=\operatorname{rank}\left(\begin{array}{cccc}
O & O & B_{1}^{-1} & O \\
O & A_{4}^{\sharp} & O & O \\
B_{1}^{-1} & O & O & O \\
O & O & O & O
\end{array}\right) \\
& =2 r+\operatorname{rank}\left(A_{4}^{\sharp}\right) .
\end{aligned}
$$

Since $\operatorname{rank}(M)=2 r+\operatorname{rank}\left(A_{4}\right), \operatorname{rank}(X)=\operatorname{rank}(M)$, thus $X=M^{\sharp}$ by Lemma 2.2.

Example 3.5. Let $M=\left(\begin{array}{cc}A & B \\ B & O\end{array}\right)$, where $A=\left(\begin{array}{cc}1 & 1+i \\ i & 0\end{array}\right)$ and $B=$ $\left(\begin{array}{cc}i & i \\ 0 & 0\end{array}\right), i=\sqrt{-1}$

By computation, $B^{\sharp}$ exists, $B A B^{\pi}=O$ and $B^{\sharp}=\left(\begin{array}{rr}-i & -i \\ 0 & 0\end{array}\right)$.

From Theorem 3.4, $M^{\sharp}$ exists, $U=\left(\begin{array}{rr}0 & -i \\ 0 & i\end{array}\right), V=\left(\begin{array}{rr}0 & 0 \\ -i & -i\end{array}\right)$ and $-B^{\sharp} A B^{\sharp}$ 
$=\left(\begin{array}{rr}1+i & 1+i \\ 0 & 0\end{array}\right)$, then $M^{\sharp}=\left(\begin{array}{rrrr}0 & -i & 0 & 0 \\ 0 & i & -i & -i \\ -i & -i & 1+i & 1+i \\ 0 & 0 & 0 & 0\end{array}\right)$.

Theorem 3.6. Let $M=\left(\begin{array}{cc}A & B \\ B & O\end{array}\right)$, where $A, B \in K^{n \times n}$. Suppose that $B^{\sharp}$ exists and $B^{\pi} A B=O$. Then

(i) $M^{\sharp}$ exists if and only if $\left(B^{\pi} A\right)^{\sharp}$ exists.

(ii) If $M^{\sharp}$ exists, then

$$
M^{\sharp}=\left(\begin{array}{cc}
U & B^{\sharp} \\
V & -B^{\sharp} A B^{\sharp}
\end{array}\right),
$$

where

$$
\begin{aligned}
& U=\left(B^{\sharp}\right)^{2} A B^{\pi}-\left(B^{\sharp}\right)^{2} A B^{\pi} A\left(B^{\pi} A\right)^{\sharp}+\left(B^{\pi} A\right)^{\sharp} ; \\
& V=-B^{\sharp} A\left(B^{\sharp}\right)^{2} A B^{\pi}+B^{\sharp} A\left(B^{\sharp}\right)^{2} A B^{\pi} A\left(B^{\pi} A\right)^{\sharp}-B^{\sharp} A\left(B^{\pi} A\right)^{\sharp}+B^{\sharp} .
\end{aligned}
$$

Proof. The proof is similar to that of Theorem 3.4.

Acknowledgments: The authors would like to thank the referee for his/her helpful comments.

\section{REFERENCES}

[1] A. Ben-Israel, T.N.E. Greville. Generalized inverses: Theory and applications. Second ed., Wiley, New York, 1974, Springer-Verlag, New York, 2003.

[2] K.P.S. Bhaskara Rao. The theory of generalized inverses over commutative rings. Taylor and Francis, London and New York, 2002.

[3] R. Bru, C. Coll, N. Thome. Symmetric singular linear control systems. Appl. Math. Letters, 15:671-675, 2002.

[4] C. Bu, J. Zhao, J. Zheng. Group inverse for a Class $2 \times 2$ block matrices over skew fields. Applied Math. Comput., 24:45-49, 2008.

[5] C. Bu, J. Zhao, K. Zhang. Some results on group inverses of block matrices over skew fields. Electronic Journal of Linear Algebra, 18:117-125, 2009.

[6] C. Bu, M. Li, K. Zhang, L. Zheng. Group inverse for the block matrices with an invertible subblock. Appl. Math. Comput., 215:132-139, 2009.

[7] C. Bu, K. Zhang, J. Zhao. Some results on the group inverse of the block matrix with a sub-block of linear combination or product combination of matrices over skew fields. Linear and Multilinear Algebra, to appear, DOI: 10.1080/03081080903092243.

[8] C. Cao. Some results of group inverses for partitioned matrices over skew fields. J. of Natural Science of Heilongjiang University, 18(3):5-7, 2001 (in Chinese). 
[9] C. Cao, X. Tang. Representations of the group inverse of some $2 \times 2$ block matrices. International Mathematical Forum, 31:1511-1517, 2006.

[10] N. Castro-González, E. Dopazo. Representations of the Drazin inverse of a class of block matrices. Linear Algebra Appl., 400:253-269, 2005.

[11] S.L. Campbell. The Drazin inverse and systems of second order linear differential equations. Linear and Multilinear Algebra, 14:195-198, 1983.

[12] S.L. Campbell, C.D. Meyer. Generalized inverses of linear transformations. Dover, New York, 1991 (Originally published: Pitman, London, 1979).

[13] S.L. Campbell, C.D. Meyer, N.J. Rose. Application of the Drazin inverse to linear systems of differential equations with singular constant cofficients. SIAM J. Appl. Math., 31:411425, 1976.

[14] M. Catral, D.D. Olesky, P. Van Den Driessche. Group inverses of matrices with path graphs. Electronic Journal of Linear Algebra, 17:219-233, 2008.

[15] J. Chen, Z. Xu, Y. Wei. Representations for the Drazin inverse of the sum $P+Q+R+S$ and its applications. Linear Algebra Appl., 430:438-454, 2009.

[16] X. Chen, R.E. Hartwig. The group inverse of a triangular matrix. Linear Algebra Appl., 237/238:97-108, 1996.

[17] D.S. Cvetković-Ilić, J. Chen, Z. Xu. Explicit representations of the Drazin inverse of block matrix and modified matrix. Linear and Multilinear Algebra, 57:355-364, 2009.

[18] C. Deng. A note on the Drazin inverses with Banachiewicz-Schur forms. Appl. Math. Comput.,213: 230-234, 2009.

[19] C. Deng, Y. Wei. A note on the Drazin inverse of an anti-triangular matrix. Linear Algebra Appl., 431:1910-1922, 2009.

[20] N. Castro-González, E. Dopazo, J. Robles. Formulas for the Drazin inverse of special block matrices. Applied Math. Comput., 174:252-270, 2006.

[21] D.S. Cvetković-Ilić. A note on the representation for the Drazin inverse of $2 \times 2$ block matrices. Linear Algebra Appl., 429:242-248, 2008.

[22] R. Hartwig, X. Li, Y. Wei. Representations for the Drazin inverse of $2 \times 2$ block matrix. SIAM J. Matrix Anal. Appl., 27:757-771, 2006.

[23] R.E. Hartwig, J.M. Shoaf. Group inverse and Drazin inverse of bidiagonal and triangular Toeplitz matrices. Austral. J. Math., 24(A):10-34, 1977.

[24] G. Heinig. The group inverse of the transformation $\varphi(X)=A X-X B$. Linear Algebra Appl., 257:321-342, 1997.

[25] S. Kirkland, M. Neumann. On group inverses of M-matrices with uniform diagonal entries. Linear Algebra Appl., 296:153-170, 1999.

[26] X. Li, Y. Wei. A note on the representations for the Drazin inverse of $2 \times 2$ block matrices. Linear Algebra Appl., 423:332-338, 2007.

[27] C.D. Meyer, N.J. Rose. The index and the Drazin inverse of block triangular matrices. SIAM. J. Appl. Math., 33:1-7, 1977.

[28] G. Wang, Y. Wei and S. Qian. Generalized inverse: theory and computations. Science Press, Beijing, 2004.

[29] Y. Wei, H. Diao. On group inverse of singular Toeplitz matrices. Linear Algebra Appl., 399:109-123, 2005. 